\title{
Correction to: Scheme for implementing dichotomic quantum measurements through non-ideal Stern-Gerlach setup
}

\author{
Arnab Adhikary · Soumik Ghosh
}

Published online: 25 June 2018

(C) Chapman University 2018

\section{Correction to: Quantum Stud.: Math. Found. https://doi.org/10.1007/s40509-018-0168-8}

The value of the non-ideal parameter $\alpha$ has been incorrectly stated to be between 0 and 1 after derivation of the time evolution of the corrupt Gaussian distribution in Section 6.2. Actually, $\alpha$ can take any value as seen in Fig. 3 and Table 3 where $\alpha$ is 3.5.

In Table 3, the phrase "non-Gaussian" is used to denote the corrupt Gaussian distribution. They refer to the same waveform.

In Section 7, before Eq. 36, the inner product is between wave functions of two opposite spins spread over the screen in the $x-z$ plane. There was incorrect formatting of the line.

The original article can be found online at https://doi.org/10.1007/s40509-018-0168-8.

A. Adhikary $\cdot$ S. Ghosh $(\bowtie)$

Department of Electronics and Telecommunication Engineering, Jadavpur University, Kolkata 700 032, India

e-mail: ghoshsoumik14@gmail.com 